\title{
The controller placement problem or the controller selection problem?
}

\author{
Keshav Sood*, Yong Xiang \\ School of Information Technology, Deakin University, Melbourne 3215, Australia \\ * Corresponding author, Email: ksood@deakin.edu.au
}

\begin{abstract}
In SDN, the control logic of packet processing devices is moved onto the SDN centralized controller. This decoupled networking architecture creates some critical concerns when compared to traditional distributed architectures. One primary concern in this architecture is the placement of the controller, which is commonly known as the CPP. From our extensive literature review, we identify that there is no strict placement rule that best fits every network in SDN. Dynamic addition and deletion of controllers is inevitable. Motivated from this, in this paper, we attempt to transform the CPP into a CSP. We show that, in the dynamic SDN environment, researchers need to pay attention to CSP issues. The clear advantage of CSP over CPP is that, in the dynamic SDN ecosystem, we can answer fundamental questions about the control plane performance, i.e., minimum number of controllers, their workload distribution, and placement/locations. Furthermore, we can meet strict, application-specific, QoS constraints.
\end{abstract}

Keywords: software-defined network, controller placement problem, flow balancing in SDN, quality of service, distributed networks, future networks

Citation: K. Sood, Y. Xiang. The controller placement problem or the controller selection problem? [J]. Journal of communications and information networks, 2017, 2(3): 1-9.

\section{Introduction}

Traditional computer networks do not scale well and are inadequate to fulfill the needs and requirements of the current Internet environment. In order to address this, SDN (Software-Defined Network), which represents a transformation from traditional switching to a new programmable switching technology, was proposed. SDN is considered as a tectonic shift in the networking industry and the anticipation come a degree of over-hype. The concept of SDN is substantially different from that of the traditional switching architectures.

In SDN principles, the control logic of data plane elements, such as switches and routers, etc. is shifted onto the control plane elements, like physical and logical SDN controllers ${ }^{[1]}$. This simplifies the management of complex networks and brings about many other significant advantages. However, this decoupling of planes adversely affects the control plane performance, and their associated issues such as latency, scalability, and availability (of the decoupled SDN architectures) are largely unstudied ${ }^{[2]}$. This decoupled and centralized architecture almost affects almost every aspect of network performance. The switch-controller distance imposed fundamental limitations on the controller availability and response time or flow-setup time for flows/packets, especially in $\mathrm{WANs}^{[3]}$. Owing to the limited capacity of handling flows by software-based controllers for handling flows, it is very challenging to respond to events in real-time, or it is difficult for controllers to behave 
in advance in both active and passive SDN operations. To alleviate these concerns, it is essential to study the performance characteristics of the controllers, and then the overall control plane.

To quantify the performance concerns of the control plane, Heller et al. investigated a new direction, called CPP (Controller Placement Problem) ${ }^{[3]}$. This perspective narrowed down the focus and addressed two vital questions: i) how many controllers are needed? and ii) where in the topology should they go? ${ }^{[3]}$. In their study, the authors proposed the concept of the CPP issue, and quantified the impact of controller placement on real topologies. They used controller-switch latency, as the key metric to determine the number of controllers and their placement. The aim of the study is to determine the number of controllers ( $K$ or $S$ ), and to minimize average latency. In other words ensuring that $\left|S^{\prime}\right|=K$ or $S$ and $L_{\text {avg }}\left(S^{\prime}\right)$ are minimum alleviates the fundamental limit on availability and convergence time.

Yao et al. pointed out that authors of Ref. [3] did not consider the factor of load in CPP work. In Ref. [4] a new algorithm, was proposed that took into account the load factor in the optimization of the number of controllers and reduced controller overloading. Furthermore, authors in Refs. $[5,6]$ focused on the same issue and determined that the diverse network needs and the physical network characteristics played a significant role in determining the number of controllers that are required. A few other researchers have investigated the same issue from different perspectives. The authors of Refs. [7,8] analyzed the resource management aspect of the CPP issue. Further, energy aware placement is analyzed in Ref. [9]. QoS (Quality of Service) guaranteed CPP is formulated by Ref. [6] and references therein. This study aims to obtain the minimum number of controllers provided the application latency delay must not exceed the application's specific QoS requirement, and their location in the network.

After thorough and careful observation, we identify the following key points about the problem from the existing research:

- The underlying topology is not fixed and is al- ways dynamic.

- The traffic behavior and size changes frequently; therefore, resources at the control plane should be incorporated accordingly.

- Rather than analyzing the controller capacity, researchers must analyze the controller's responsiveness, which is the primary factor that determines end-to-end delay.

Bases on the key concerns and observations, we define the following.

Definition 1 The CPP can be transformed into the CSP (Controller Selection Problem).

In this paper, first of all, we emphasize the above three concerns in detail. Secondly, based on our observations, we propose that the CPP can be transformed into CSP by exploring the relationship between traffic intensity, resources requirement, and QoS requirement. Eventually, the aim is to set up a network with high performance ability and less CAPEX and OPEX requirements.

Our contributions in this paper are listed below:

- We show that the load level and response time of a controller affect the controller's performance when the flow setup is reactively performed. Thus, to optimize the control layer performance, the solutions must be topology-independent and adaptive to the needs of the underlying network behavior. No single solution in the dynamic SDN environment best fits all scenarios with respect to controller placement.

- Based on the above observations, we propose that a CPP be transformed into a CSP. Furthermore, we proposes a topology independent framework to optimize the control layer with the objective of calculating the optimal number of controllers to reduce the workload, and investigate the placement/location of the controllers.

Our aim is not to determine the optimal placement of controllers in the network, but to motivate the CSP. The rest of the article is organized as follows. Section 2 provides preliminaries and section 3 surveys related work. In section 4 , we discuss motivational examples emphasizing why CSP is preferred over CPP. Analysis and discussion are presented in 
section 5. Section 6 highlights some potential selection metrics of our proposal, and we conclude the paper in section 7 .

\section{Preliminaries}

SDN was originally developed by Stanford scientists to conduct experiments and support innovation with new protocols. OpenFlow protocol used in SDN encouraged vendors to implement and enable SDN in switches and other networking products ${ }^{[10]}$. The SDN switching mechanism, as shown in Fig. 1, comprises four steps. In the basic operation, the SDN switch analyze every incoming flow to find a matching tuple/flow from the flow-table. Depending on this, it takes the required necessary action such as broadcast, unicast, dropping the flow, etc. (Matching was based on 10 tuple till 2008, but is based on 32 tuple from protocol version v.1.5 onwards). In the absence of any match, the switch forwards the packet to the SDN controller for instruction on how to deal with packet ${ }^{[1,11]}$. The centralized controller populates the switch with flow table entries. We encourage readers to read Refs. [1,2] thoroughly and see references therein for better understanding.

\section{Related works}

To optimize the control layer performance, researchers of Ref. [3] considered the distance between controller and switch as the performance metric. This enabled minimization of the end-toend delay, and computation of the optimal number of controllers. However, the proposed solution was topology-dependent and the work load factor on controller was not considered in depth. In Ref. [7], the authors considered average flow request among switches and switch-to-controller, and proposed heuristic algorithm to dynamically shift the controller load. In their research effort authors did not provide the control flow traffic statistics.

Further, Ghazisaeedi et al. ${ }^{[12]}$ showed that HTTP applications in the Internet covered approximately
$47.7 \%$ of the Internet traffic. They proposed rerouting of traffic using OpenFlow switches to improve resources utilization. A Plug- $n$-Server solution was proposed in Ref. [13] to analyze the network status and assign client requests with the aim of minimizing the controller's response time. Wang et al. ${ }^{[14]}$ proposed use of wild card rules in the matching tuple to reduce the work load on the controller. Researchers also proposed the deployment of distributed controllers as a general approach to achieve scalability. However, the challenges like deriving the optimal number of controllers, their placement in the network, and load distribution still remain ${ }^{[15,16]}$.

Sallahi et al. proposed a new mathematical model for $\mathrm{CPP}$ in SDN. For a given set of switches, the proposed model determines the optimal number, location, and type(s) of controllers. The authors also attempted to minimize the network element's cost ${ }^{[17]}$. We observe that the load factor is only partially considered in this study. Further, the researchers used one optimizer to evaluate the model, and optimizers use different bound algorithms. Thus, adding multiple factors may affects the accuracy of the model.

Authors of Ref. [18] proposed a non-zero sum based game theoretic scheme to derive the optimal number of controllers and their mapping to switches. The idea is simple and cost-effective, and operates in real-time. However, no QoS improvements are critically evaluated in this paper. QoS guaranteed controller placement is proposed by Cheng et al. ${ }^{[6]}$, and three algorithms were proposed and tested on real world datasets. Initially, the number of switches that must be connected to a controller is calculated by using incremental algorithm. However, we identify that this is not always accurate as the traffic rate send out by switches varies. Recently, we proposed a distributed decision scheme framework of the proposed modules in SDN controller. We attempt to investigate the feasibility and benefits of the proposed flow-balancing solution, which considers network dependencies such as resources, performance, and cost simultaneously ${ }^{[16]}$. However, this scheme does not consider the priority of flows which we addresses in this paper. 
1. Usually the first packet miss the flow-table entry.

2. The flow forwarded to controller in order to obtain a new-flow table so that a required action on the particular flow can be performed.

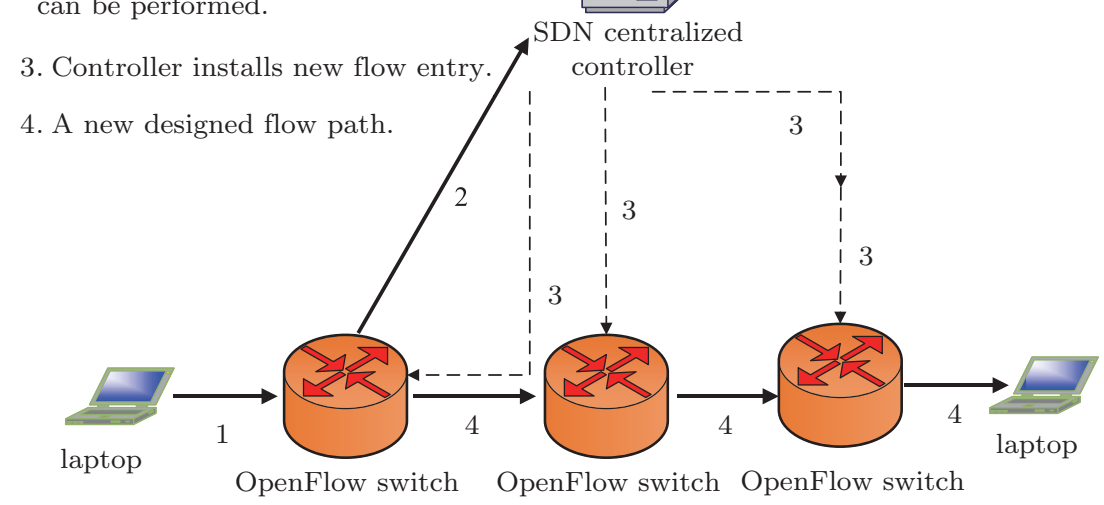

Figure 1 The basic work-flow of SDN

\section{Motivating examples}

In this section, we discuss the reasons behind our main three concerns based on which we prefer the CSP problem over CPP problem.

The underlying topology is not fixed and is always dynamic. Tootoonchian et al. ${ }^{[19]}$ found that adding switches beyond a threshold reduces the overall controller's throughput. However the packet-in rate from switches is not predictable. The incoming traffic pattern is hard to predict, therefore, the data out rate of switches differs, which sometimes exceeded the threshold handling capacity. This adversely affects the control plane performance. Furthermore, it is possible that, in case of failures or to provide high availability, we need to change the network topology. Thus, the solution based on fixed network topologies may not be viable completely on the new topology.

The traffic behavior and size changes frequently; therefore, resources at the control plane should be incorporated accordingly. The response time of controller and delay is important in case of dynamic variations in load conditions at the controller. In this case, it is very challenging to meet the strict QoS demand of different traffic models ${ }^{[20]}$. Therefore, in order to minimize traffic delay, it is important that the controller response time must be minimal. High availability and maintenance of low response time is a necessary requirement. This needs multiple controllers in the network. Addition and deletion of controllers based on the workload and maintenance of performance is vital.

Rather than analyzing the controller capacity, researchers must analyze the controller's responsiveness, which is the primary factor to alleviate endto-end delay ${ }^{[3,19]}$. From the above two points it is clear that the most important factor is the end-toend delay. We can compromise the factors such as the cost, resources, etc. but not the performance (latency and throughput). Thus, the focus should be on minimizing the controller's response time to flows rather than evaluating the controller's capacity.

In a nutshell, no single solution is a best fit for all cases. Thus, a simple, adaptive, topologyindependent and low complexity real-time solution is required. Dynamic addition or deletion of virtual machine based controllers based on the traffic demand and performance requirements is essential.

\section{Analysis and discussion}

The expensive load balancers at the SDN controllers must be capable to alleviate a set of fundamental 
concerns. This includes optimization (minimization) of the number of controllers required, their workload, and placement in the network. To address this concern, researchers ${ }^{[3]}$ adopted $K$-Centre and $K$-Median theories. For the optimal number of controller, $S^{\prime}$, and their placements, authors determined the average case latency between the controller and switch. In graph theory, $G(V, E)$, propagation delays are represented by edge weights, $d(v, s)$ represents the shortest path from node $v \in V$ to $s \in V$, and the number of nodes is $n=|V|$. The aim is to determine the $K$ or $S$ number of controllers, such that the average latency can be minimized, i.e., $\left|S^{\prime}\right|=K$ or $S$ and $L_{\text {avg }}\left(S^{\prime}\right)$ is minimum.

$$
L_{\mathrm{avg}}\left(S^{\prime}\right)=\frac{1}{n} \sum_{v \in V} \min _{s \in S^{\prime}} d(v, s) .
$$

The above equation is applicable only when the load condition on the controller is static. The reason is that the number of controllers $S$ or $K$ and their respective position change as the network load condition changes dynamically ${ }^{[16,18]}$. Therefore, we observe that solving one vital concern has a clear and straight impact on various other network performance parameters. Therefore, the logical addition or deletion of controllers is vital as load condition changes.

First, we show that as the service rate and packet sending rate of switches changes, the performance of the controller changes accordingly. The relationship between flow-setup time and number of $s$ switches is explored by using the following equation ${ }^{[21]}$. We performed Matlab simulations to perform the following three analysis.

$$
T(t)=\frac{s+1}{2(\mu-\lambda s)}
$$

Using this equation, we obtained the plot shown in Fig. 2 which visualizes the relationship between controller flow-setup time and number of $s$ switches from one controller. The set values of service rate $(\mu)$ and packet arrival rate $(\lambda)$ pps are approximately near the real-world statistics provided by Ref. [21].

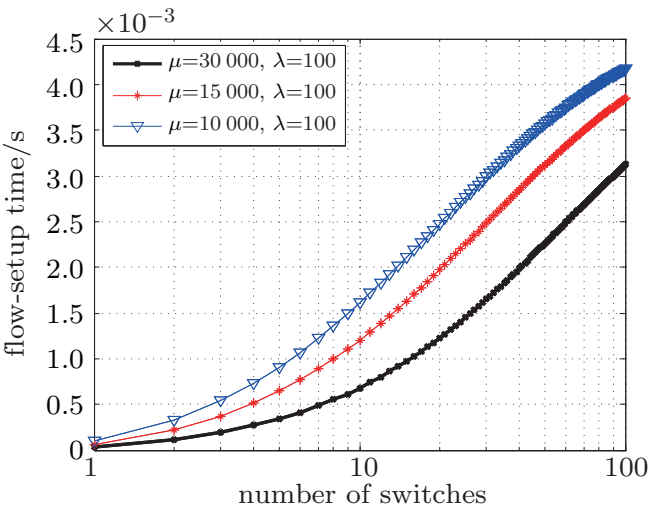

Figure 2 Relationship between $\mu$ and the number of switches $s$ with regards to flow-setup time

In the first analysis, it can be seen that the controller can manage approximate 80 switches and the maximum flow-setup time is $1.5 \mathrm{~ms}$ for $\mu=10000$ at fixed $\lambda=100$. In contrast, the controller's capacity to manage 80 switches changes accordingly at $\mu=30000$, i.e., the flow-setup time to manage the switches (at same data rate) changes as $\mu$ varies.

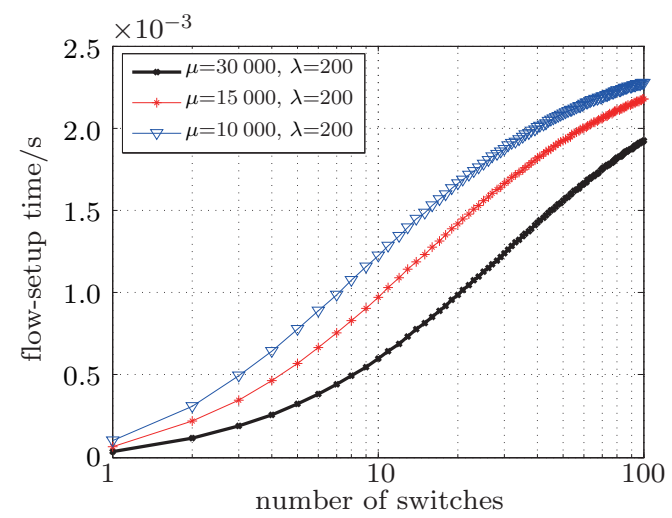

Figure 3 Flow-setup time relationship with $\mu$ and the number of switches

In the second analysis, we change the data rate (200 packet/s) of switches and observe the flow-setup time for different values of $\mu$. Comparing Fig. 2 and Fig. 3, we see that, at $\mu=10000$, the controller's capacity to manage switches changes.

From this analysis, we observe that the controller's capacity affects the application's QoS requirements considerably. As a result, the number of underlying switches must be limited to a certain bound 
so that QoS requirements of an application can be guaranteed; further, this necessitates dynamic flowbalancing. This reveals that, as the packet arrival rate increases, the capacity of the controller varies accordingly, and it becomes necessary to add new controllers in the network, and vice-versa. For better QoS management and decrease end-to-end delay, it is essential the network resources must be incorporated adaptively. Adding more number of resources helps to reduce the flow-setup time, which eventually helps to improve the QoS requirements, as shown in Fig. 4.

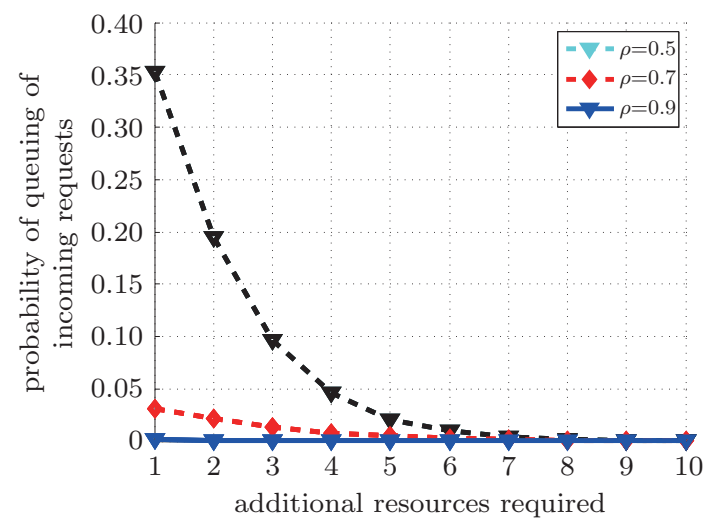

Figure 4 Relationship between $p_{\text {queueing }}$ and additional resources required $(m)$, at a given traffic intensity, $\rho=\frac{\lambda}{\mu}$, for system stability $0 \leqslant \rho \leqslant 1$

In the third analyses, we showed that deploying additional resources or controllers in the network decreases the probability of flows in the queue. Addition or deletion of VM controllers helps optimize the resource requirement, while maintaining the QoS, provided the condition $T(t) \leqslant \Delta T$ is satisfied. Here, $T(t)$ represents the controller's response time to flows and $\Delta T$ is the QoS requirement of a specific flow. The probability that new incoming flow has to wait in the queue is given by

$$
p[\text { queuing }]=p_{0} \frac{(m \rho)^{m}}{m !(1-\rho)}=1-\sum_{k=0}^{m} p_{0} \frac{(m \rho)^{k}}{k !} .
$$

Here, $p_{0}$ is a constant that is equals to $1-\frac{\lambda}{\mu}$ and $k \geqslant 0$. The key equations are taken from classical $M / M / 1$ and $M / M / m$ models $^{[22]}$.

We observe that the probability of flows in the queue decreases, as shown in Fig. 4, when there are more resources deployed. This helps to minimize the response time to flows. Furthermore, we observed that adding and deleting VM controllers helps optimize the resource requirements. With regard to physical controllers, they can be put into ideal/sleep mode by means of some optimization method.

These results give insights that the load determination and traffic offloading based only on packet-in events at controller may not completely valid; thus, flow-based traffic diversion approaches need to be investigated. Next, we propose a flow-based traffic diversion mechanism framework in distributed SDN networks, as shown in Fig. 5.

In this method, once a flow request arrives at the controller, the "prioritize incoming flows" engine prioritizes each flow according to its QoS requirement. Mice and elephant flows can be broadly categories in the same way. After ranking flows according to the priority, the controller decides where the request with high priority should be served to satisfy the application specific QoS requirements, locally or on another controller. If it is decided to served on another controller, the request will be forwarded to the controller that satisfy the application specific QoS requirement. We assume that the time delay for forwarding is negligible. Further, we assume that SDNi message is used for interconnectivity and exchange of messages among peer controllers. Here, interSDN communication module is used to communicate with peer controllers and the connected underlying switches. The state collection control function collects and update the peer controller network states.

It is necessary to mention that the logical addition and deletion of controllers is mandatory and based on the dynamic workload demand and QoS requirement of the flow. It is easy to invoke and revoke the number of logical controllers using our method. However, researchers emphasize that switching physical controllers off is complicated; thus, they may be put into sleep/ideal mode.

Our method involves mapping flow onto a controller that can satisfy the flow QoS requirements. Thus, rather than solving a placement issues, our 


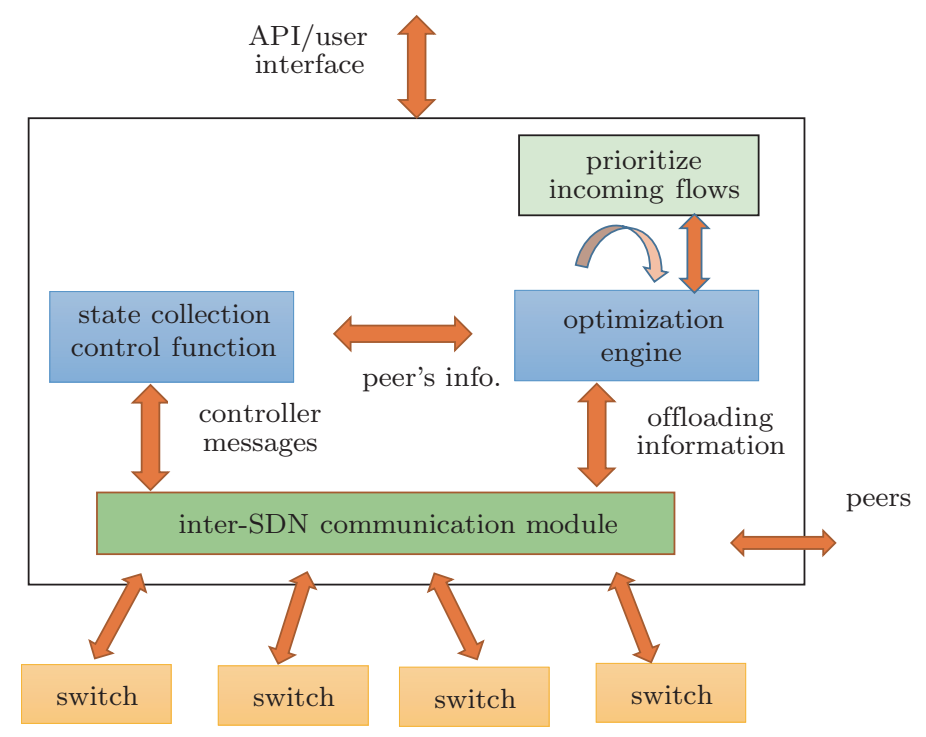

Figure 5 The proposed per flow based weighted routing modules of the SDN controller

method transforms the CPP problem into a CSP problem based on the QoS. Various other objective functions can be added to determine how the flow will select the controller; this is out of the scope of this paper. For now, we just select the controller based on QoS requirement of flow.

\section{Potential selection metrics}

Controller selection strategies can be designed based on the individual network needs. In this section, we highlight key dominant factors in networking that motivate design policies for the selection of the controller. Such policies can be placed either at the centralized management controller, end-node (user device such as mobile, laptop, etc.), or a gateway can be used for this purpose.

Some key factors are described next. The energy efficiency and sustainability of resources is gaining importance in networking as a factor that motivate the management of a large number of resources in an efficient way. CSP policies can help in this context. CSP algorithms can be proposed for power management and virtualization in SDN-based cloud models to optimize the network's operation and infrastructure. Observability, controllability, and predictability are very critical challenges in networking.
Real-time monitoring and controlling for the collection of precise energy consumption data can be proposed. With such optimization goals, the controllers or SDN nodes can intelligently adjust their energy consumption.

Many other factors can be used in CSP. We welcome the community to contribute and propose novel CSP algorithms. This is very early work in SDN and we believe that research in this domain help ease the control layer workload as well aid in the faster adoption of SDN. The potential selection metrics or high level view of examples will motivate researchers to design novel and flexible forwarding and selection decision-based algorithms that could greatly enhance the business versatility.

\section{Conclusion and future work}

SDN represents a tectonic shift in the networking industry. So far, studies have only focused on the potential of this new paradigm shift, and not much is understood about the limitations of this new technology model. From our literature review, we observed that very less has been published in-line with the work shown in this paper. In this research effort, we proposed that the $\mathrm{CPP}$ can be transformed into a CSP. In CPP, it is very hard to find the minimum 
as well as optimal number of controllers, their workload and placement. The reason is that the dynamic nature of the network traffic changes frequently. We showed that, as traffic pattern changes, the reactive flow setup time for the controllers change. Therefore, rather than focusing on a fixed placement strategy, the application must be mapped onto the controller which can process the application within its bounded QoS requirements.

In the future, we plan to built a theoretical model for the CSP, incorporating multiple parameters such as delay, number of resources, energy, CPU utilization, etc. The current investigation is based on classical queuing models. We will investigate some models that gives a closed form or approximation results to this issue. Furthermore, we will design a prototype of this concept so that appropriate evaluation can be executed and demonstrated. For in-depth investigations big data streams can be included in this work to validate the "priority function" in the given framework. Apart from these future works, investigations toward designing a strategy to achieve energy-cost reduction can be a potential venue for research.

\section{References}

[1] X. Xia, Y. Wen, C. H. Foh, et al. A survey on softwaredefined networking $[\mathrm{J}]$. IEEE communications surveys and tutorials, 2015, 17(1): 27-51.

[2] I. F. Akyildiz, A. Lee, P. Wang, et al. A roadmap for traffic engineering in sdn-openflow networks [J]. Computer networks, Elsevier, 2014, 71:1-30.

[3] H. Heller, R. Sherwood, N. McKeown. The controller placement problem [C]//First Workshop on Hot Topics in Software-Defined Networks, Helsinki, Finland, 2012: 7-12.

[4] G. Yao, J. Bi, Y. Li, et al. On the capacitated controller placement problem in software defined networks [J]. IEEE communications letters, 2014, 18(8): 13391342 .

[5] Y. Jimenez, C. C. Pastor, A. J. Garcia. On the controller placement for designing a distributed SDN control layer $[\mathrm{C}] / /$ Proceedings of Networking Conference (IFIP 2014), Trondheim, Norway, 2014: 1-9.

[6] T. Y. Cheng, M. Wang, X. Jia. QoS-guaranteed controller placement in SDN [C]//IEEE Global Communication Conference (GLOBECOM), San Diego, USA,
2015: 1-6.

[7] S. Lange, S. Gebert, T. Zinner, et al. Heuristic approaches to the controller placement problem in large scale SDN networks [J]. IEEE trans. netw. serv. manag., 2015, 12(1): 4-17.

[8] Y. H. Zhou, M. F. Zhu, L. M. Xiao, et al. A load balancing strategy of sdn controller based on distributed decision $[\mathrm{C}] / /$ 13th IEEE International Conference on Trust, Security, and Privacy in Computing and Communications (TrustCom), Beijing, China, 2014: 851-856.

[9] R. Rivera, A. K. W. Chin, S. Soh. GreCo: an energy aware controller association algorithm for software defined networks [J]. IEEE communications letters, 2015, 19(4): 541-544.

[10] N. McKeown, T. Anderson, H. Balakrishnan, et al. OpenFlow: enabling innovation in campus networks? [J]. Newsletter ACM SIGCOMM computer communication review, 2008, 38(2): 69-74.

[11] S. Sezer, S. Scott-Hayward, P. K. Chouhan, et al. Are we ready for SDN? Implementation challenges for softwaredefined networks $[\mathrm{J}]$. IEEE communications magazine, 2013, 51(7): 36-43.

[12] E. Ghazisaeedi, R. Tafazolli. Mobile core traffic balancing by OpenFlow switching system [C]//IEEE Globecom Workshops, Atlanta, USA, 2013: 824-829.

[13] N. Handigol, S. Seetharaman, M. Flajslik, et al. Plug- $n-$ Server: load balancing web traffic using OpenFlow [J]. Demo at ACM SIGCOMM, 2009.

[14] R. Wang, D. Butnariu, J. Rexford. OpenFlow-based server load balancing gone wild $[\mathrm{C}] / / 11$ th USENIX Conference on Hot Topics in Management of Internet, Cloud, and Enterprise Networks and Services, Boston, USA, 2011: 12-12.

[15] K. Sood, S. Yu, Y. Xiang, et al. Control layer resource management in SDN-IoT networks using multiobjective constraint [C]//IEEE 11th Conference on Industrial Electronics and Applications (ICIEA), Hefei, China, 2016: 71-76.

[16] K. Sood, S. Yu, Y. Xiang, et al. A general QoS aware flow-balancing and resource management scheme in distributed software-defined networks $[\mathrm{J}]$. IEEE access, 2016, 4: 7176-7185

[17] A. Sallahi, M. St-Hilaire. Optimal model for the controller placement problem in software defined networks [J]. IEEE communications letters, 2015, 19(1): 30-33.

[18] H. K. Rath, V. Revoori, S. M. Nadaf, et al. Optimal controller placement in software defined networks (SDN) using a non-zero-sum game [C]//IEEE 15th International Symposium World of Wireless, Mobile and Multimedia Networks (WoWMoM), Sydney, Australia, 2014: 1-6.

[19] A. Tootoonchian, S. Gorbunov, Y. Ganjali, et al. On controller performance in software-defined networks [C]//2nd USENIX Workshop on Hot Topics in Management of Internet, Cloud, and Enterprise Networks and Services, San Jose, USA, 2012: 10-10. 
[20] B. Briscoe, A. Brunstrom, A. Petlund, et al. Reducing Internet latency: a survey of techniques and their merits [J]. IEEE communications surveys and tutorials, 2016, 18(3): 2149-2196.

[21] L. Yao, P. L. Hong, W. Zhou. Evaluating the controller capacity in software-defined networking $[\mathrm{C}] / / 23 \mathrm{rd}$ International Conference on Computer Communication and Networks (ICCCN), Shanghai, China, 2014: 1-6.

[22] L. Kleinrock. Queueing system. vol. I: theory [Z]. Wiley Interscience, 1975.

\section{About the authors}

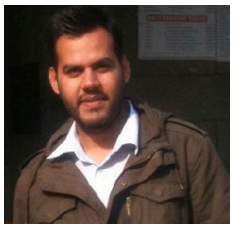

Keshav Sood [corresponding author] worked with Terminal Ballistic Research $\mathrm{Lab}$ as a trainee. He did his B.Tech in electronics engineering with distinction and M.Tech degree in optical fibre engineering. Currently he is towards his $\mathrm{PhD}$ degree in School of IT at Deakin University, Melbourne, Australia. He is a recognised professional engineer by Engineers
Australia. He is also a student member of IEEE. He served as TPC member of various IEEE conferences includes IEEE INFOCOM, IEEE Bigdata service, and IEEE ITNAC. His current research domain are network security and flow management in SDN. (Email: ksood@deakin.edu.au)

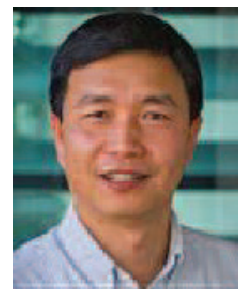

Yong Xiang (SM'12) received the Ph.D. degree in electrical and electronic engineering from The University of Melbourne, Australia. He is a professor and the director of the Artificial Intelligence and Image Processing Research Cluster, School of Information Technology, Deakin University, Australia. His research interests include signal and system estimation, information and network security, multimedia (speech/image/video) processing, and wireless sensor networks. He has published more than 110 refereed journal and conference papers in these areas. He is an associate editor of IEEE Signal Processing Letters and IEEE Access. He has served as program chair, TPC chair, symposium chair, and session chair for a number of international conferences. (Email: yxiang@deakin.edu.au) 